\title{
Hypervelocity runaways from the Large Magellanic Cloud
}

\author{
D. Boubert, ${ }^{1 \star}$ D. Erkal, ${ }^{1}$ N. W. Evans ${ }^{1}$ and R. G. Izzard ${ }^{1}$ \\ ${ }^{1}$ Institute of Astronomy, University of Cambridge, Madingley Road, Cambridge CB3 OHA, UK
}

Accepted XXX. Received YYY; in original form ZZZ

\begin{abstract}
We explore the possibility that the observed population of Galactic hypervelocity stars (HVSs) originate as runaway stars from the Large Magellanic Cloud (LMC). Pairing a binary evolution code with an N-body simulation of the interaction of the LMC with the Milky Way, we predict the spatial distribution and kinematics of an LMC runaway population. We find that runaway stars from the LMC can contribute Galactic HVSs at a rate of $3 \times 10^{-6} \mathrm{yr}^{-1}$. This is composed of stars at different points of stellar evolution, ranging from the main-sequence to those at the tip of the asymptotic giant branch. We find that the known B-type HVSs have kinematics which are consistent with an LMC origin. There is an additional population of hypervelocity white dwarfs whose progenitors were massive runaway stars. Runaways which are even more massive will themselves go supernova, producing a remnant whose velocity will be modulated by a supernova kick. This latter scenario has some exotic consequences, such as pulsars and supernovae far from star-forming regions, and a small rate of microlensing from compact sources around the halo of the LMC.
\end{abstract}

Key words: Magellanic Clouds - binaries: general - stars: kinematics and dynamics

\section{INTRODUCTION}

In this decade of large and precise kinematic datasets, it is tempting to go hunting for outliers. These range from the unusual 30-40 $\mathrm{km} \mathrm{s}^{-1}$ OB-runaways, first explained by Blaauw (1961) as the runaway former companions of supernova progenitors, to the extreme $>500 \mathrm{~km} \mathrm{~s}^{-1}$ hypervelocity stars (HVSs), which are unbound from the Milky Way (MW). The latter are suspected to have been accelerated by the Hills mechanism, where the tidal disruption of a binary by the massive black hole (SMBH) Sgr A* in the Galactic Centre results in the rapid ejection of one of the stars (Hills 1988). Alternative explanations for these stars include dynamical ejection from young clusters (Perets 2009), extreme supernova runaway scenarios (Portegies Zwart 2000), tidal debris from an accreted dwarf galaxy (Abadi et al. 2009) or an SMBH in the Large Magellanic Cloud (Boubert \& Evans 2016).

In this work, we explore the consequences of the production of runaway stars in the Large Magellanic Cloud (LMC). The LMC has a star-formation rate of $0.2 \mathrm{M}_{\odot} \mathrm{yr}^{-1}$ (Harris \& Zaritsky 2009) and an orbital velocity of $378 \mathrm{~km} \mathrm{~s}^{-1}$ (van der Marel \& Kallivayalil 2014), so it is plausible that runaway stars from the LMC could contribute a meaningful proportion of the Galactic HVSs. More massive galaxies typically

^ E-mail: d.boubert,nwe,derkal,rgi@ast.cam.ac.uk have more of everything (e.g. globular clusters and supernova), but this does not necessarily include having more unbound, escaping stars. This is because lowering the mass of the galaxy lowers the required escape velocity. As most stellar processes which produce high velocity stars have a steeply decreasing distribution with increasing velocity, it follows that decreasing the mass of a galaxy can result in more escaping stars. The velocities produced by these processes are set by stellar properties, and these are only weakly dependent on the host galaxy. Some other possible origins of unbound LMC stars include stripping from the LMC by a previous passage of the SMC (Besla et al. 2013), formation in the gas of the leading arm of the LMC (Casetti-Dinescu et al. 2014), and ejection from the LMC either by a putative SMBH in the centre of the LMC (Edelmann et al. 2005; Boubert \& Evans 2016) or dynamical interactions in a stellar cluster, possibly involving an intermediate mass black hole (Gualandris \& Portegies Zwart 2007).

The field of fast-moving stars is beset by a muddle of nomenclature, which stems from the difference between classifying stars by how fast they are moving or by their origin. Among HVSs this is a crucial distinction. A star may be ejected by the Hills mechanism, but remain bound to the galaxy. Conversely, a star may be unbound, but not produced by the Hills mechanism. Runaway stars are usually defined as $\mathrm{OB}$ stars with peculiar velocities in excess of $40 \mathrm{~km} \mathrm{~s}^{-1}$ (Blaauw 1961), with either dynamical ejection from a young cluster or a supernova ejecting the progenitor's 
companion as their origin. However, the slower cousins of the binary supernova runaways are also termed runaways by several authors, with increasing use of the term walkaways for those runaways ejected slower than $10 \mathrm{~km} \mathrm{~s}^{-1}$ (de Mink et al. 2012, 2014; Lennon et al. 2016). A convention sometimes used in the literature is to refer to unbound Hills stars as hypervelocity and unbound runaway stars as hyperrunaway (eg. Perets \& Šubr 2012; Brown 2015). However, this is open to the objection that it is in practice difficult to determine the origin of the known unbound stars in the Galaxy. For example, they may not originate in the Milky Way (e.g., Boubert \& Evans 2016) and - as we show in this paper they may not even originate with the Hills mechanism. To clarify the terminology of this paper, we exclusively use the term runaway to refer to stars of all velocities whose binary companion has gone supernova and the term hypervelocity to refer to stars of any origin which are unbound from the Milky Way. All stars emitted from a binary tidally disrupted by a central black hole of either galaxy are Hills stars. To avoid the confusion of referring to stars which escape the LMC as HVSs with respect to the LMC, we will use the terms LMC remainers/escapers to refer to stars which are bound/unbound to the LMC.

In Section 2, we describe the method we use to generate runaways and then follow their stellar evolution and orbit in an LMC-MW potential. There are many observables associated with runaway stars which escape the LMC and we discuss these in Section 3. Our conclusions in Section 4 are that runaway stars escaping the LMC must contribute to the Milky Way hypervelocity star population, but that the stellar types and distribution of these hypervelocity runaways are dependent on the assumed binary evolution model.

\section{LMC RUNAWAY EJECTION MODEL}

There are several ingredients required for a model of the ejection of runaway stars from the LMC. Assuming a metallicity and star formation history for the LMC, we evolve a synthetic population of single and binary stars and identify the runaway stars. The runaways are then initialised in the LMC disk and their subsequent orbits integrated through an evolving N-body potential of the LMC and the Galaxy. The outcomes of the stellar evolution of these runaway stars and their kinematics are then transformed into observable properties.

\subsection{Star Formation History of the LMC}

Our method requires knowledge of the time dependent starformation rate (SFR) and metallicity of the LMC. Harris \& Zaritsky (2009) found that the star formation rate of the LMC over the past 5 Gyr has been constant at $0.2 \mathrm{M}_{\odot} \mathrm{yr}^{-1}$ within a factor of two. However, this period of constancy was preceded by a quiescent epoch between 5 and 12 Gyr ago. We thus assume a constant star formation rate over the entire $1.97 \mathrm{Gyr}$ we simulate.

Piatti \& Geisler (2013) investigated the age-metallicity relation for the LMC using photometry across 21 fields. They derived an approximate scaling,

$[\mathrm{Fe} / \mathrm{H}]=C+\left(\frac{\partial[\mathrm{Fe} / \mathrm{H}]}{\partial t}\right) t+\left(\frac{\partial[\mathrm{Fe} / \mathrm{H}]}{\partial a}\right) a$, with $C=-0.55 \pm 0.02 \mathrm{dex}, \partial[\mathrm{Fe} / \mathrm{H}] / \partial t=-0.047 \pm$ $0.003 \mathrm{dex} \mathrm{Gyr}^{-1}$ and $\partial[\mathrm{Fe} / \mathrm{H}] / \partial a=-0.007 \pm 0.006$ dex degree ${ }^{-1}$, where $a$ is the de-projected angular distance from the centre of the LMC. The dependency on the angular distance is argued by Piatti \& Geisler (2013) to be negligible, because under the assumption of an LMC distance of $50 \mathrm{kpc}$

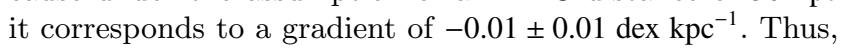
we assume a constant metallicity throughout the LMC starforming regions. Over the $1.97 \mathrm{Gyr}$ of our simulations, even the temporal gradient is mostly negligible, producing a change in $[\mathrm{Fe} / \mathrm{H}]$ of $-0.093 \pm 0.006 \mathrm{dex}$. Thus, we form stars at a constant metallicity of $Z=0.008$.

Most stars form in clusters (Lada \& Lada 2003), but this does not mean that star formation in the LMC is clumpy. The currently most prominent star-forming region in the LMC is 30 Doradus, also known as the Tarantula Nebula. De Marchi et al. (2011) found the star formation rate to be of the order $200 \mathrm{M}_{\odot} \mathrm{Myr}^{-1}$ over at least the last $30 \mathrm{Myr}$ for objects in the mass range $0.5-4.0 \mathrm{M}_{\odot}$. This is consistent with the more recent work of Cignoni et al. (2015) who, as part of the Hubble Tarantula Treasury Project, found that the star formation rate (SFR) in 30 Doradus has exceeded the average LMC SFR for the last 20 Myr. While 30 Doradus is one of the most active star formation regions in the Local Group, comparing its rate $200 \mathrm{M}_{\odot} \mathrm{Myr}^{-1}$ to the rate for the entire LMC $0.2 \mathrm{M}_{\odot} \mathrm{yr}^{-1}$ reveals that 30 Doradus makes up only $0.2 \%$ of the recent star formation activity of the LMC. We are thus well justified in forming stars directly proportional to the density of the assumed LMC disk potential and neglecting any inhomogeneities due to star forming clusters. We note that if this assumption does break down, it would reveal itself as a skewed density distribution of the ejected stars on the sky. This is because the location from which runaway stars are ejected is encoded in the velocity of those runaways through the contribution of the LMC disk rotation at the location of ejection.

\subsection{Binary Evolution}

A standard prescription for the distribution of runaway star ejection velocities $v_{\mathrm{ej}}$ is an exponential law in the form $\exp \left(-v_{\mathrm{ej}} / v_{\mathrm{s}}\right)$, where $v_{\mathrm{s}} \approx 150 \mathrm{~km} \mathrm{~s}^{-1}$ is a characteristic velocity which sets the width of the distribution (used by Bromley et al. 2009 and Kenyon et al. 2014 who matched to binary star simulations of Portegies Zwart 2000). However, this velocity distribution is simplistic because the highest ejection velocities require close binaries. Close binaries interact, making the ejection velocities of runaways a sensitive function of the binary initial conditions. Given that the magnitude and colour of stars can be thought of broadly as a proxy for their mass and that one of the most important parameters in binary interaction is the ratio of masses $q$, the colour and ejection velocity of a runaway star must be interdependent.

We form stars in bursts every 1 Myr. This is driven by a computational consideration to allow for a simple implementation of star formation in which we sample single and binary stars from analytic distributions until we have formed the required mass of stars. A SFR of $0.2 \mathrm{M}_{\odot} \mathrm{yr}^{-1}$ means we are thus forming starburts with a total mass of $2 \times 10^{5} \mathrm{M}_{\odot}$. Only a small fraction of this mass is used to form runaways. Our model population consists of both single stars and binaries, but no higher-order multiples are considered. To gen- 
erate the population, we sample in the primary mass and, for binaries, in the mass ratio and initial period. We sample systems one-by-one until we have formed the required total mass of stars in a timestep.

We first sample the primary mass of each system from the Kroupa (2001) IMF,

$$
N\left(M_{1}\right) \propto \begin{cases}M_{1}^{-0.3}, & \text { if } 0.01<M_{1} / \mathrm{M}_{\odot}<0.08, \\ M_{1}^{-1.3}, & \text { if } 0.08<M_{1} / \mathrm{M}_{\odot}<0.5, \\ M_{1}^{-2.3}, & \text { if } 0.5<M_{1} / \mathrm{M}_{\odot}<80.0, \\ 0, & \text { otherwise. }\end{cases}
$$

We calculate the binary fraction as a function of primary mass. Arenou (2010) provides an analytic empirical fit to the observed binary fraction of various stellar masses,

$F_{\text {bin }}\left(M_{1}\right)=0.8388 \tanh \left(0.079+0.688 M_{1}\right)$.

We validate this formula by comparing to the data of Raghavan et al. (2010), who provide the binary fraction as a function of spectral type. The binary fraction has only been well studied in the Milky Way and it is possible that the lower metallicity stars in the LMC could exhibit a different dependency on the primary mass. Our results turn out not to be overly dependent on the exact form assumed for the dependency of the binary fraction on the primary mass. This is because in our grid of evolved binary systems most runaways come from high-mass systems in which the binary fraction is close to unity in all prescriptions. We assume a flat mass-ratio distribution for each system over the range $0.1 \mathrm{M}_{\odot} / M_{1}<q<1$. The period distribution is taken from Duquennoy \& Mayor (1991) and is a normal distribution in $\log _{10}(P /$ days $)$ with a mean of 4.8 and a standard deviation of 2.3 , truncated to lie between -2.0 and 12.0. The observed period distribution of OB-type stars is closer to being loguniform (Opik 1924; Sana et al. 2012), however the error incurred by this choice is subdominant to the uncertainty in the outcome of the common-envelope phase.

We model the properties of stars ejected from binary systems in which one component goes supernova using the BINARY_C population-nucleosynthesis framework (Izzard et al. 2004, 2006, 2009). BINARY_C is based on the binarystar evolution (BSE) algorithm of Hurley et al. (2002) updated to include nucleosynthesis, wind-Roche-lobe-overflow (Abate et al. 2013, 2015), stellar rotation (de Mink et al. 2013), accurate stellar lifetimes of massive stars (Schneider et al. 2014), dynamical effects from asymmetric supernovae (Tauris \& Takens 1998), an improved algorithm describing the rate of Roche-lobe overflow (Claeys et al. 2014) and corecollapse supernovae (Zapartas et al. 2017). In particular, we take our black hole remnant masses from Spera et al. (2015), use a fit to the simulations of Liu et al. (2015) to determine the impulse imparted by the supernova ejecta on the companion and assume that the natal kick on the comapct remnants of Type II supernovae is Maxwellian (Hansen \& Phinney 1997), all of which were options previously implemented in BINARY_C. We use version 2.0pre22, SVN 4585. Grids of stars are modelled using the BINARY_GRID2 module to explore the single-star parameter space as a function of stellar mass $M$, and the binary-star parameter space in primary mass $M_{1}$, secondary mass $M_{2}$ and orbital period $P$.

The initial conditions of the binaries sampled are compared to a binary grid and we identify all runaways which are formed by this population. We pre-compute this binary grid of 8,000,000 binaries with primary mass $M_{1}$, mass ratio $q$ and period $P$ having the ranges,

$$
\begin{aligned}
& 8.0 \leq M_{1} / \mathrm{M}_{\odot} \leq 80.0, \\
& 0.1 \mathrm{M}_{\odot} / M_{1} \leq q \leq 1,
\end{aligned}
$$$$
-2.0 \leq \log _{10}(P / \text { days }) \leq 12.0 \text {. }
$$

The distribution of runaway ejection velocity $v_{\mathrm{ej}}$ and $B-V$ colour at the time of ejection from the progenitor binary is shown in Fig. 1. A discussion of the detailed structure in the data is elsewhere (Boubert et al, in prep.) but the distribution can be divided into two regions. The slower runaways with $v_{\mathrm{ej}}<30 \mathrm{~km} \mathrm{~s}^{-1}$ are the classical runaways in which the progenitor binary does not interact prior to the supernova. Conversely, runaways with $v_{\mathrm{ej}}>30 \mathrm{~km} \mathrm{~s}^{-1}$ are those whose progenitor binary did interact. When the primary evolves to the giant branch, it overflows its Rochelobe onto the companion, provided the companion is sufficiently close (De Marco \& Izzard 2017). Mass transfer from a higher mass star to a lower mass star shrinks the binary orbit and increases the rate of mass transfer. This process is self-reinforcing and leads to common envelope evolution and further shrinkage of the binary. If the common envelope is dissipated before the stars merge, the binary is left in a close orbit. When the primary does go supernova shortly afterwards the natal kick on the remnant may be sufficient to unbind this close binary. In this case the rapid orbital velocity of the companion prior to the explosion results in a fast runaway. The impulse of the supernova ejecta impacting on the companion can contribute to the ejection velocity, but for almost all the runaways considered this was a negligible effect. The structure in this plot simply reflects the different channels that this behaviour can follow, together with the dependency on the mass and evolutionary state of the companion. The sideways chevron with $v_{\mathrm{ej}}=400-800 \mathrm{~km} \mathrm{~s}^{-1}$ and $B-V \sim 0$ corresponds to cases in which the companion is so massive initially that the binary is close to being equal-mass. When a more massive star transfers mass to a lower-mass companion, the orbit shrinks. The converse is that when a less massive star transfers mass to a higher mass companion, the orbit grows. Thus, sustained mass transfer causes the companion to first approach and then retreat from the primary. The fastest runways are those in which the stars are closest prior to the common-envelope phase and thus the tip of the chevron represents systems in which the binary is equal-mass prior to the common-envelope.

The binary origin of the runaway stars which escape the LMC influences their subsequent evolution because prior to ejection more than $90 \%$ experience mass transfer from the primary. The transferred mass can be up to several $\mathbf{M}_{\odot}$ in extreme cases. Thus the runaways in our simulation would appear as blue stragglers in comparison to their progenitor population, i.e. would be bluer than a single star of equivalent age and mass. If the age of a candidate runaway star is estimated using single star isochrones and is compared to a flight time from the LMC they may be discrepant, because the rejuvenation of the star by mass transfer prior to ejection may have extended the lifetime of the star by a few 100 Myr.

A finite but non-negligible time elapses between the formation of a binary and the ejection of a runaway (Zapartas 


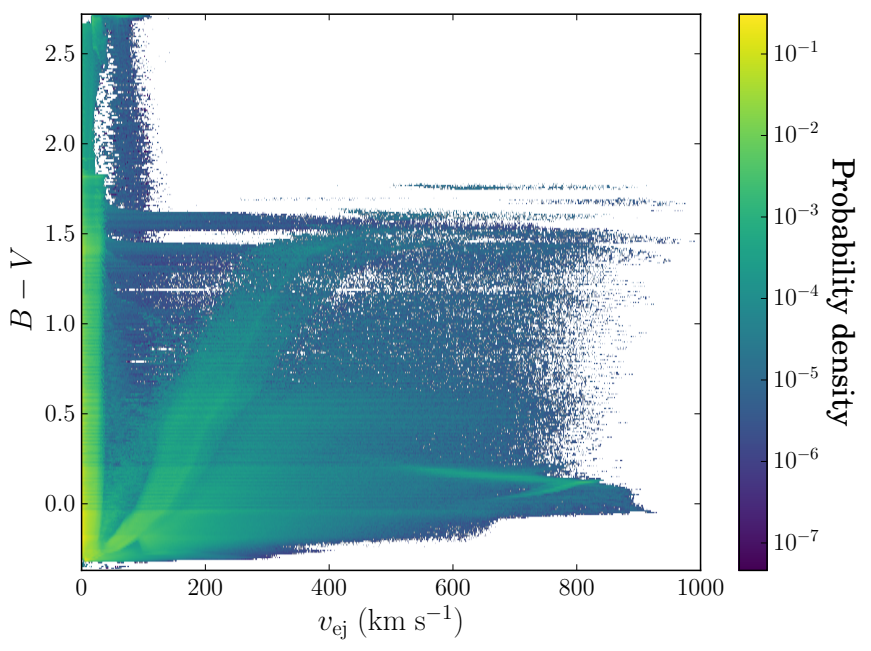

Figure 1. Probability density distribution in velocity-colour space at the time of ejection from the progenitor binary of the runaways produced by our binary evolution grid assuming LMC metallicity $Z=0.008$ and common-envelope ejection efficiency $\alpha_{\mathrm{CE}}=1.0$.

et al. 2017), typically between 1-50 Myr. We bin the emission time of our runaways to the nearest $10 \mathrm{Myr}$ because that is the frequency of snapshots in the N-body simulation. Once we have the time of ejection, we evolve each system which produces a runaway to the present day to ascertain the current observable properties. We record the stellar type, the mass, the Johnson-Cousins UBVRIJHK magnitudes, the Sloan Digital Sky Survey ugriz magnitudes and the Gaia $G$, $G_{\mathrm{BP}}, G_{\mathrm{RP}}$ and $G_{\mathrm{RVs}}$ magnitudes. Because most of our binaries are B-type stars evolved on Gyr timescales, more than than $70 \%$ of our runaways cease nuclear burning before the present day. If there is a supernova, we record the time that it occurred so we can later extract its location from the Nbody model. We sample from a Maxwellian distribution of kick velocities for the neutron star and black hole remnants of Type II supernova progenitors (discussed in more detail in Section 3.5) and run a second N-body integration to compute the final location of these compact remnants.

\section{$2.3 \quad$ N-body MW/LMC Model}

To model the runaways produced by the LMC, we use an $N$-body simulation of the LMC and the Milky Way galaxies. The LMC is modelled with two components (disk and dark matter halo) while the Galaxy is modelled with three components (disk, bulge and dark matter halo). The initial conditions are chosen such that the relative position of the Galaxy to the LMC matches their present day value within $2 \sigma$ (see Sec. 4 of Mackey et al. 2016 for more details on the simulations). Our simulations are evolved with the $\mathrm{N}$-body part of GADGET-3. This is similar to GADGET-2 (Springel 2005 ) but modified in two critical ways. First, we track the location of the centre of mass of the LMC by using a shrinking sphere algorithm on the inner $10 \mathrm{kpc}$ at each timestep. As a consistency check, the potential minimum of LMC particles is computed every $49 \mathrm{Myr}$ and we find no significant jumps in the LMC position. Second, the code is modified to release massless tracer particles with a given offset in position and velocity from the LMC. These are used to model the runaways. Before injecting any tracers, the simulation is evolved for 1.97 Gyr to the present and we record the LMC disk rotation curve, radial density profile, vertical density profile, orientation, position and velocity as a function of time. Fits to these properties, along with the extra velocity components of runaways (described below), are used to generate the initial conditions for the final simulation in which, as the LMC evolves in time, tracer particles are released representing the runaways.

The velocity vector of stars ejected from the LMC has three major components: the orbital velocity of the LMC, the rotation of the LMC disk and the ejection velocity of the runaway. The velocity is dominated in most cases by the $378 \mathrm{~km} \mathrm{~s}^{-1}$ orbital velocity of the LMC (van der Marel \& Kallivayalil 2014). We initialise our runaways by sampling in cylindrical coordinates $(R, z, \phi)$ with a weighting factor $\rho(R, z, \phi)$ which accounts for the density of the LMC disk at each location. From the N-body simulation, we find distributions of the tangential, radial and vertical velocities of the stars in the LMC disk at each point in the disk and at various times spaced at $10 \mathrm{Myr}$. We sample in these to determine the location and velocity of the progenitor binary at the moment of the supernova. We then add the ejection velocity by multiplying the ejection speed with a randomly-oriented unit vector. The position and velocity are then converted into the rest frame of the Galaxy.

Runaways are initialised in the simulation as massless particles every $10 \mathrm{Myr}$ as described in Section 2.2 and their orbits integrated to the present day. It is important to note that we sample in a large number of parameters and the number of generated runaways is relatively small. Thus, the extreme outliers of our population are subject to smallnumber statistical uncertainties.

\subsection{Observables}

We calculate heliocentric observables for each of our runaways by assuming that the Sun is at $R_{\odot}=8.5 \mathrm{kpc}$ and the Milky Way's disk rotation speed $v_{\text {disk }}=240 \mathrm{~km} \mathrm{~s}^{-1}$ with a solar peculiar velocity of $\left(U_{\odot}, V_{\odot}, W_{\odot}\right)=(11.1,12.24,7.25) \mathrm{km} \mathrm{s}^{-1}$ (Schönrich et al. 2010). We define those stars whose present location is $20 \mathrm{kpc}$ from the LMC to have escaped the LMC. This is similar to the observed $22.3 \pm 5.2 \mathrm{kpc}$ tidal radius of the LMC (van der Marel \& Kallivayalil 2014). A subset of the LMC escapers will also be hypervelocity with respect to the Milky Way. We define stars which are Galactic HVSs to be those with a Galactic rest frame velocity greater than

$$
\begin{aligned}
v_{\mathrm{esc}}(x) & =\left(624.9-9.41543 x+0.134835346 x^{2}-1.292640 \times 10^{-3} x^{3}\right. \\
& \left.+6.5435315 \times 10^{-6} x^{4}-1.3312833 \times 10^{-8} x^{5}\right) \mathrm{km} \mathrm{s}^{-1}
\end{aligned}
$$

where $x=r / 1 \mathrm{kpc}$ and $r$ is the spherical Galactocentric radius. We take this escape velocity curve from Brown et al. (2014) who calculated it for a three-component potential which approximates sufficiently well our live Milky Way Galaxy. We then take the magnitudes from Section 2.2, redden them using the Schlegel et al. (1998) dust map and correct them to heliocentric apparent magnitudes. We use the present day Cartesian coordinates of the stars to calculate the heliocentric kinematic observables of each star including 


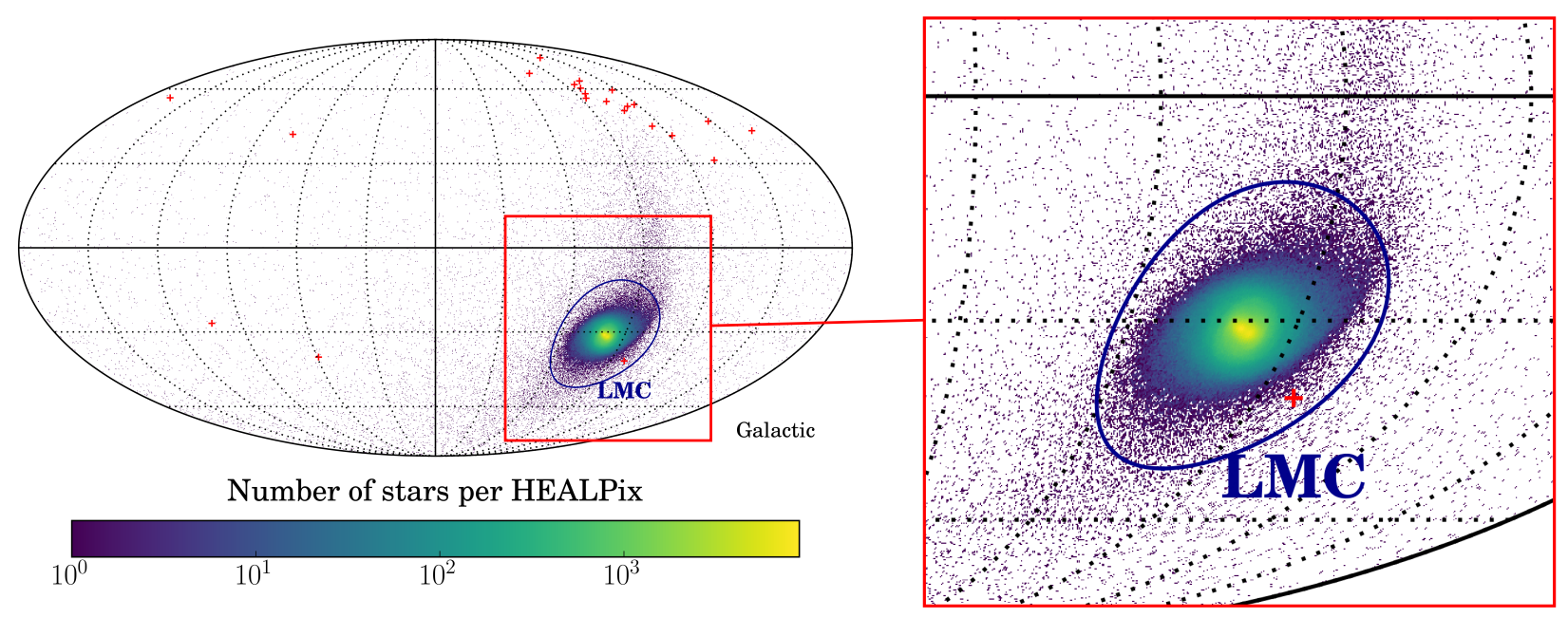

Figure 2. Left: All-sky present day distribution of runaways produced by our model of the LMC. The blue circle corresponds to the assumed tidal radius of the LMC of $20 \mathrm{kpc}$. The red crosses are the observed population of B-type HVSs. Right: Zoom-in at higher resolution to illustrate the structure of our LMC disk. An animation of the evolution of this plot through each snapshot of our simulation is available at https://youtu.be/eE-1JXBP1J8.

equatorial coordinates, distance, line-of-sight velocity and proper motions.

\section{PROPERTIES OF LMC RUNAWAYS}

The natural consequence of binary evolution in the LMC is a population of runaway stars with extreme properties. In our simulation, tens of thousands of stars escape the LMC with thousands surviving as main-sequence stars at the present day. Their spatial properties and kinematics are discussed in Section 3.1. If the LMC is as massive as recent work suggests (e.g. Kallivayalil et al. 2013; Peñarrubia et al. 2016; Jethwa et al. 2016) and as is assumed in our orbital integration, then the LMC is only marginally bound to the Galaxy and is on its first pericentre passage. A significant fraction of the stars which are LMC escapers are also unbound from the Galaxy, and so are HVSs. We discuss this possibility and compare to the known population of HVSs in Section 3.2. Existing observations of a number of populations of stars in the outskirts of the LMC lend indirect evidence to our hypothesis, as outlined in Section 3.3. The prospects for detecting an escaping LMC runaway population are discussed in Section 3.4. Lastly, a substantial fraction of our runaway stars go supernova resulting in a host of more exotic observables which we consider in Section 3.5. These include Type II supernovae far out in the LMC halo, pulsars tens of kiloparsecs from the nearest site of recent star formation and microlensing by compact remnants.

\subsection{Spatial Distribution and Kinematics}

The most notable feature is the extreme anisotropy of the LMC runaway distribution on the sky, which is aligned along the orbit of the LMC (Fig. 2). The stars we see at a particular point on the sky are a combination of stars which were ejected slowly a long time ago and stars which were ejected rapidly but more recently. The orbit of the LMC varies in heliocentric distance and so a magnitude-limited survey will miss both low-mass recent ejections and highmass, high-velocity runaways that have travelled far enough to be beyond the detection limit. We find a range of stellar types for both LMC escapees and Milky Way HVSs (Table 1). At the present day, most of our runaways are remnants which reflects the skew in the runaway mass distribution introduced by the preference for high mass primaries to host high mass companions. The lower HVS fraction of white dwarfs is because these are the remnants of the more massive of our runaways and higher mass stars are, to first order, ejected at lower velocities. This can be shown by considering the simple case of a circular binary where, if the mass of the primary and the separation are held constant, the orbital velocity of the secondary $v_{2}$ only exhibits a dependency on the total mass of the system $M$ through $v_{2} \propto M^{-1 / 2}$. Increasing the mass of the secondary thus decreases its orbital velocity, which in most cases is the dominant contributor to the ejection velocity. The lack of helium white dwarfs is to be expected. Helium white dwarfs can only be formed if the ignition of helium can be avoided, and therefore they can only be produced from the evolution of low-mass stars over a Hubble time or if a more massive star has its hydrogen envelope stripped by a companion (e.g. Althaus \& Benvenuto 1997). Because we specifically consider the scenario in which the companion escapes after the explosion of the primary, the companion does not have a chance to evolve to the giant branch and then experience mass transfer. Conversely, if the companion remains bound to the primary post-SN, it could then experience mass-loss as it evolves. Observed counterparts of this channel are the well known pulsar - helium white dwarf binaries (e.g. Backer 1998). The observed single, low-mass, helium white dwarfs are instead thought to be the remnants of giant-branch donor stars whose envelope was stripped when their companion exploded as a SN Ia (Justham et al. 2009).

The orbit of the LMC is close to being polar and thus the Galactic latitude of an LMC runaway star approximately determines its kinematics. In Fig. 3, we plot the kinematics of the predicted LMC runaway population against Galactic 


\begin{tabular}{lrrr}
\hline \hline Type & LMC remainers & LMC escapers & MW HVSs \\
\hline LM-MS & 86577 & 1227 & $77.1 \%$ \\
MS & 245486 & 7485 & $65.2 \%$ \\
HG & 1112 & 31 & $64.5 \%$ \\
GB & 1753 & 79 & $76.0 \%$ \\
CHeB & 23533 & 487 & $66.7 \%$ \\
EAGB & 678 & 12 & $83.3 \%$ \\
TPAGB & 320 & 8 & $75.0 \%$ \\
HeMS & 15 & 0 & - \\
HeHG & 2 & 0 & - \\
HeGB & 0 & 0 & - \\
HeWD & 0 & 0 & - \\
COWD & 510338 & 5233 & $57.0 \%$ \\
ONeWD & 202206 & 436 & $43.3 \%$ \\
NS & 146323 & 398527 & $82.9 \%$ \\
BH & 162646 & 445562 & $83.0 \%$ \\
\hline Total & 1380989 & 858997 & $82.6 \%$ \\
\hline \hline
\end{tabular}

Table 1. Summary of stellar types at the present day by number of stars which either remain bound to or escape the Large Magellanic Cloud, and the fraction of the latter which are hypervelocity stars with respect to the Milky Way. Key: LM-MS Low Mass Main Sequence, MS - Main Sequence, HG - Hertzprung Gap, GB - Giant Branch, CHeB - Core Helium Burning, EAGB Early Asymptotic Giant Branch, TPAGB - Thermally Pulsating Asymptotic Giant Branch, HeMS - naked Helium Main Sequence, HeHG - naked Helium Hertzsprung Gap, HeGB - naked Helium Giant Branch, HeWD - Helium White Dwarf, COWD - CarbonOxygen White Dwarf, ONeWD - Oxygen-Neon White Dwarf, NS - Neutron Star, BH - Black Hole.

latitude. We also plot the known HVSs and several observed populations of OB-type stars near the LMC which are discussed further in Sections 3.2 and 3.3, respectively.

A convenient benefit of simulating runaway stars from a galaxy is that it enables the calculation of the escape velocity curve, which at each distance from the centre of a galaxy gives the minimum speed required for a star at that location to be unbound. We take the initial velocities and radii in the frame of the $\mathrm{LMC}$ for those stars which we know subsequently escape to beyond $20 \mathrm{kpc}$ from the LMC. Because these occur sufficiently frequently at all radii within the LMC, we estimate the escape velocity by finding the curve that bounds these stars from below in the $r_{\text {init }}-v_{\text {init }}$ plane. This is complicated by the presence of stars which escape the LMC through the Lagrange points, so in practice we bin the stars radially and find the first percentile in velocity in each bin after removing outliers with $v_{\mathrm{esc}} \leq 90 \mathrm{~km} \mathrm{~s}^{-1}$. We fit a fifth order polynomial through these values and obtain,

$$
\begin{aligned}
v_{\mathrm{esc}}(x) & =\left(252.1-26.74734 x+2.44534040 x^{2}-0.164199176 x^{3}\right. \\
& \left.+6.24490163 \times 10^{-3} x^{4}-9.04817931 \times 10^{-5} x^{5}\right) \mathrm{km} \mathrm{s}^{-1},
\end{aligned}
$$

where $x=r / 1 \mathrm{kpc}$ and $r$ is the spherical radius from the LMC centre, which we plot in Fig. 4. Note that because we have a lower initial density of stars at large radii, the escape velocity curve is less accurate at these distances and we would not advocate using it outside $15 \mathrm{kpc}$. Eqn. 6 is the escape velocity curve of the LMC in isolation. The LMC has been truncated by the Milky Way at the tidal radius by the present day and thus the escape velocity currently is lower than over the previous $1.97 \mathrm{Gyr}$.

\subsection{Hypervelocity Stars (HVSs)}

If all HVSs originate in the Galactic Centre, we expect them to be isotropically distributed on the sky. However, Brown et al. (2009a) found that eight of the 14 HVSs in the Brown et al. $(2007 \mathrm{~b}, 2009 \mathrm{~b})$ targeted surveys are in the constellations of Leo and Sextans, despite the surveys covering one fifth of the sky. This anisotropy is not simply a selection effect, since Brown et al. (2007b) is $100 \%$ complete for stars with $17<g_{0}^{\prime}<19.5$ over the $7300 \mathrm{deg}^{2}$ covered by the Sloan Digital Sky Survey Data Release 6 and Brown et al. (2009b) is $59 \%$ complete for stars with $19.5<g_{0}^{\prime}<20.5$ over the same region. Brown et al. (2009a) attempted to verify the significance of the anisotropy by showing that the HVSs are clustered compared to the stars in the surveys in both Galactic latitude and longitude at $3 \sigma$ significance, in angular separations at $5 \sigma$ significance and in two-point angular correlation at $\sim 3.5 \sigma$ significance. Brown (2015) states that there is currently "no good explanation for the anisotropic distribution of unbound late B-type stars". Boubert \& Evans (2016) suggested that this anisotropy could be explained by Hills ejection of stars by a currently undetected SMBH at the centre of the LMC.

An LMC origin had previously been explored for the one HVS in the southern hemisphere, HE 0437-5439, which was discovered by Edelmann et al. (2005). The flight time is longer than the main-sequence lifetime of the star and hence either it is a blue straggler, and was ejected as a binary from the Galactic Centre, or it has its origin in the LMC. The mechanism that ejected HE 0437-5439 from the LMC has been suggested to be either interactions with a black hole more massive than $10^{3} \mathrm{M}_{\odot}$ (Gualandris \& Portegies Zwart 2007) or dynamical ejection from a cluster (Przybilla et al. 2008).

In this work, we consider the population of HVSs produced by the binary supernova runaway mechanism operating in the LMC, which Table 1 demonstrates is substantial. However, we find that our model LMC runaway HVSs which make it into the footprint of SDSS are inconsistent with the observed HVSs, being in the mass range $1.6 \mathrm{M}_{\odot}<M<3.0 \mathrm{M}_{\odot}$ rather than the $M>3.0 \mathrm{M}_{\odot}$ of the B-type HVSs. The reason for this is clear from Fig. 1. Those stars that make it into the footprint of SDSS have $v_{\mathrm{ej}} \gtrsim 200 \mathrm{~km} \mathrm{~s}^{-1}$ and there is a distinctly low probability density of runaways at these speeds with $B-V<0$. There are three possibilities that either dismiss or resolve this discrepancy:

(i) The observed B-type HVSs do originate in the Milky Way galaxy from one of the processes discussed above and the anisotropy indicates a symmetry-breaking in these processes. One example would be if the binary stars which interact with Sgr $\mathrm{A}^{*}$ are scattered from a disk in the Galactic nucleus rather than coming from a spherically-symmetric population.

(ii) The observed B-type HVSs originate in the LMC, but are ejected by a process which has a higher typical ejection velocity than runaways - either the Hills mechanism or dynamical ejection from a cluster. 


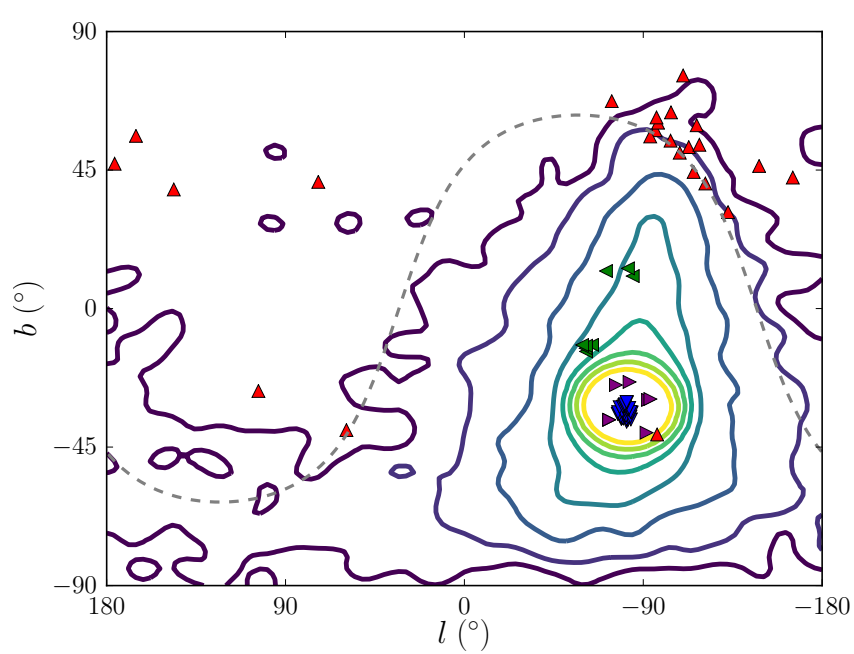

(a) Latitude - Longitude

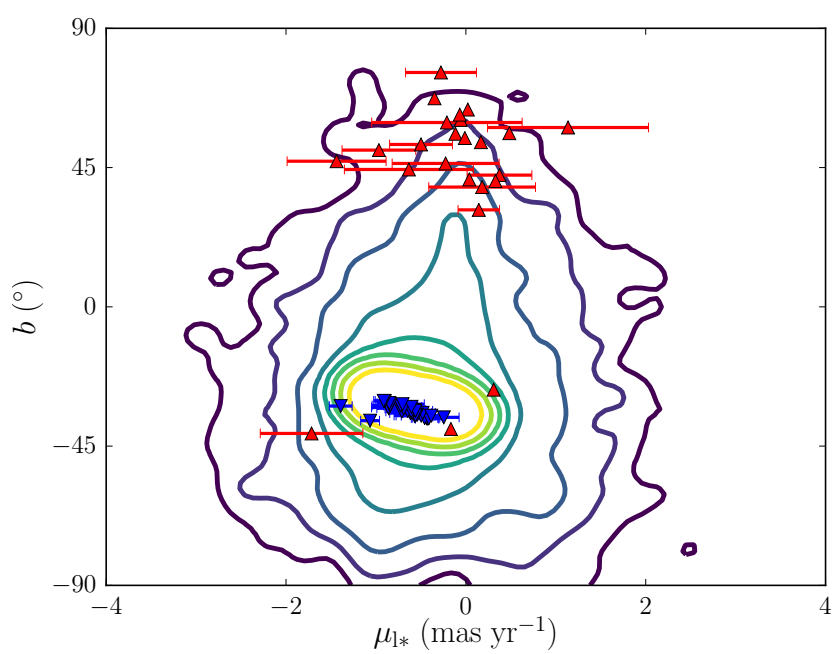

(c) Latitude - Longitudinal Proper Motion

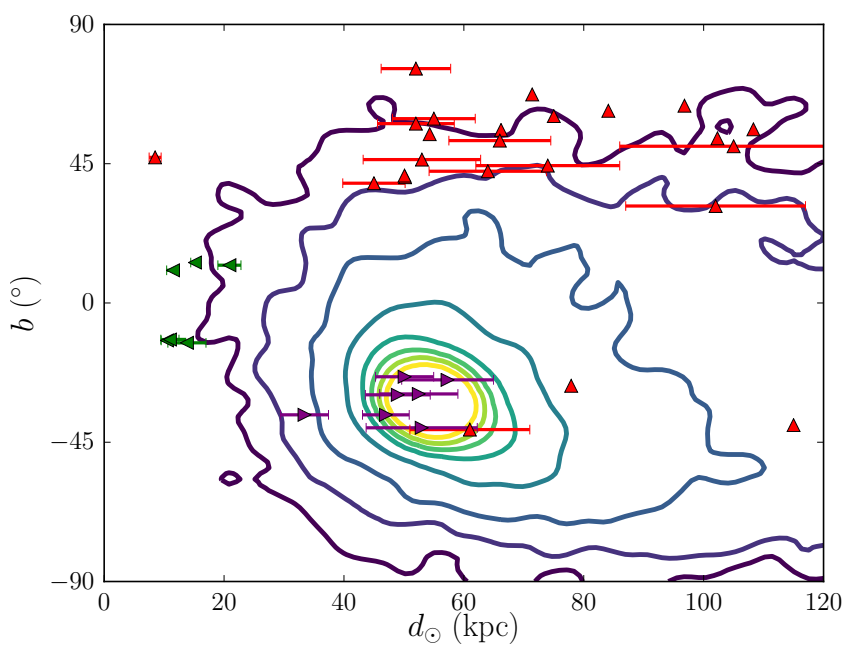

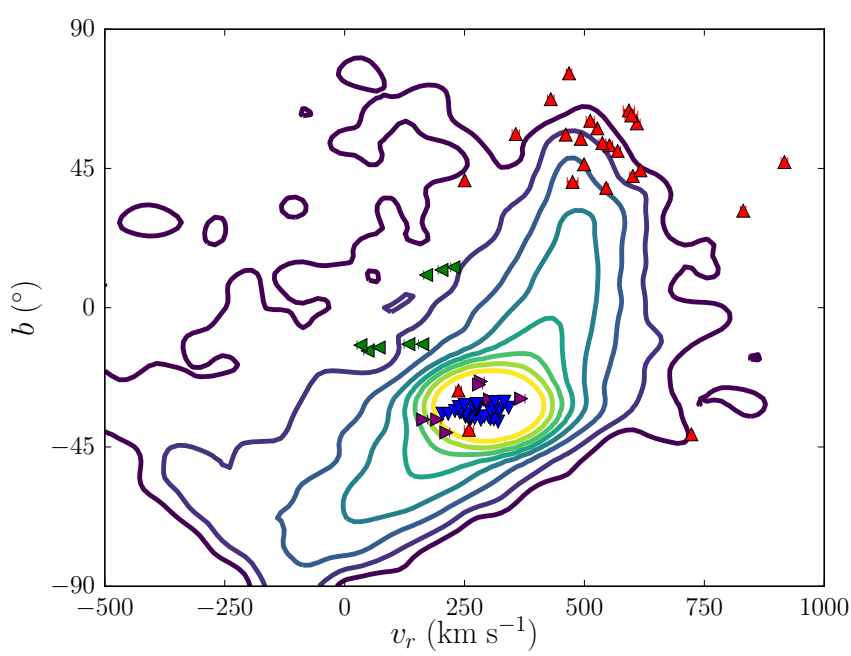

(b) Latitude - Radial Velocity

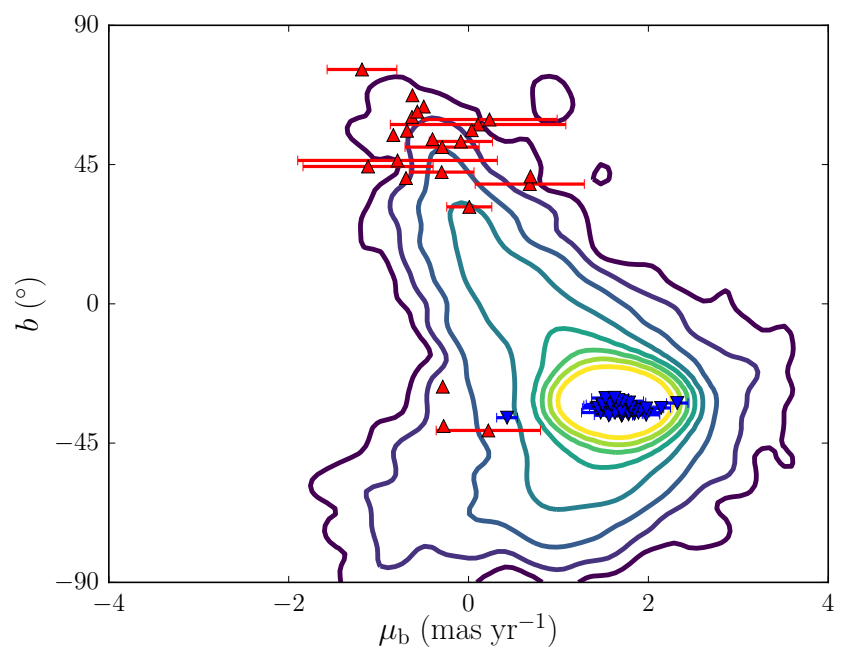

(d) Latitude - Latitudinal Proper Motion
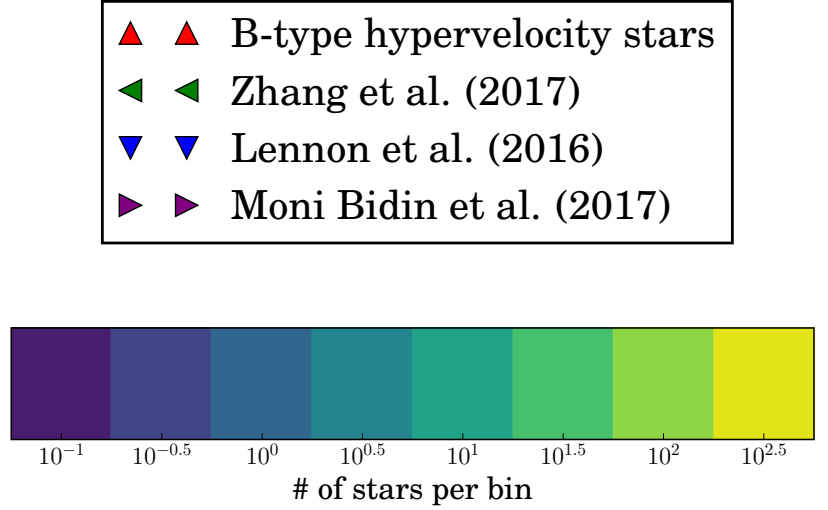

(e) Latitude - Distance

Figure 3. Predictions of the kinematics of our LMC runaway model plotted as logarithmically-spaced contours of the number of stars in each bin. The bins are defined by a $100 \times 100$ grid over the range of each plot. Also shown are observations of OB-stars near the LMC in the literature: the known B-type hypervelocity stars, stars which may have formed from the gas in the leading arm (Zhang et al. 2017), candidate runaways in the LMC (Lennon et al. 2016) and young stars in the outskirts of the LMC (Bidin et al. 2017). The distances for the stars from Zhang et al. (2017) and Bidin et al. (2017) are calculated from distance moduli, proper motions were only available for the Lennon et al. (2016) stars and a subset of the hypervelocity stars, and the Lennon et al. (2016) stars only have distances by association with the LMC. The grey dashed line marks the celestial equator and SDSS photometry only covers the region above this line.

MNRAS 000, 1-13 (2017) 


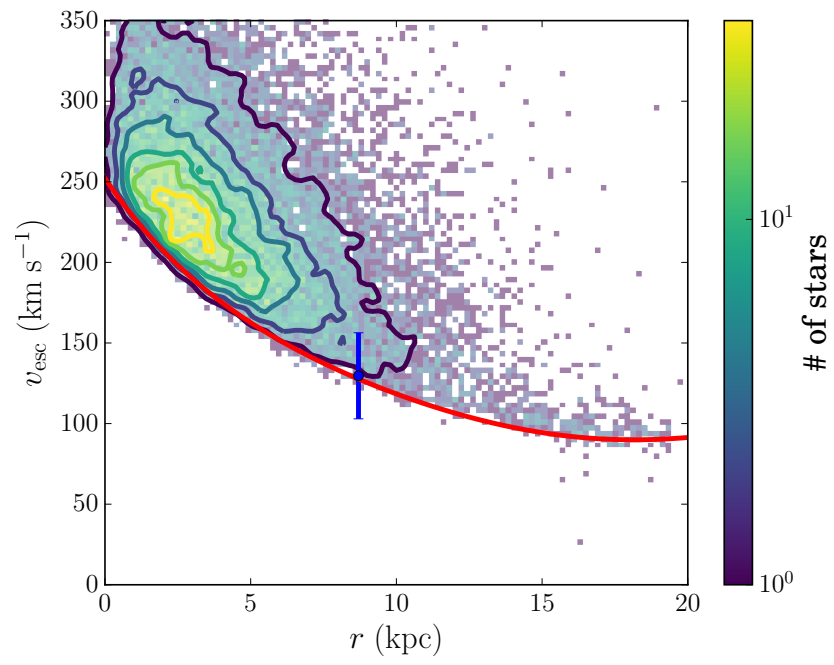

Figure 4. The escape velocity curve of our modelled LMC potential (see Section 3.1). The contours illustrate the distribution of our LMC escapers, the red line is our estimated escape velocity curve and the blue point is the mass constraint for the LMC $M(8.7 \mathrm{kpc})=(1.7 \pm 0.7) \times 10^{10} \mathrm{M}_{\odot}($ van der Marel \& Kallivayalil 2014) converted to escape velocity with $v_{\mathrm{esc}}=\sqrt{2 \mathrm{G} M / r}$, where $\mathrm{G}$ is the gravitational constant and $r$ is the spherical radius.

(iii) Our prescription for the common-envelope evolution of binary stars is inaccurate. We follow Hurley et al. (2002) and set $\alpha_{\mathrm{CE}}=1.0$, where $\alpha_{\mathrm{CE}}$ is the efficiency with which the orbital energy of the binary can be used to remove the common envelope, but this parameter is not well-constrained observationally. If we instead set $\alpha_{\mathrm{CE}}=0.1$, we find a highvelocity distribution where the HVSs would be predominantly of A and B type. There is additional uncertainty in the fraction $\lambda_{\mathrm{CE}}$ of the binding energy of the envelope which is required to eject the envelope. We use a fit to tabulated numerical results which is implemented in BINARY_C (Dewi \& Tauris 2000; Tauris \& Dewi 2001). However, the tabulated $\lambda_{\mathrm{CE}}$ were calculated at solar metallicity. The parameters $\alpha_{\mathrm{CE}}$ and $\lambda_{\mathrm{CE}}$ appear together in the $\alpha$-prescription and so it is the combination $\alpha_{\mathrm{CE}} \lambda_{\mathrm{CE}}$ which sets the post-common-envelope separation. An error in either parameter could explain the possible discrepancy between the observed HVSs and our LMC runaway model.

We can evaluate whether the observed HVSs originate in the LMC, whilst being agnostic about the mechanism, by considering the runaway stars in our model to be tracer particles of the kinematic distribution of stars ejected from the LMC. When discussing the known HVSs we specifically refer to the candidates discovered by the HVS surveys (Brown et al. 2005, 2006a, 2007a,b, 2009b, 2012, 2014) in addition to HE 0437-5439 (Edelmann et al. 2005) and US 708 (Hirsch et al. 2005), with recent updated proper motions from the Hubble Space Telescope (Brown et al. 2015). Fig. 3 demonstrates that the $6 \mathrm{D}$ kinematics of the known HVSs are consistent with the expectations for an LMC origin. The agreement in proper motions and distance is not surprising. The known HVSs were found in observation campaigns (Brown et al. 2006a,b, 2009b) that selected for distant B-type stars in the footprint of SDSS, and thus most have $\delta>0^{\circ}$ and are at distances $50<d<120 \mathrm{kpc}$. At these distances, the proper motion projects to nearly zero independent of whether the star originates in the Galaxy or LMC. It is surprising, however, that an LMC origin can reproduce the clustering in the $b-l$ and $b-v_{r}$ plots, neither of which can be explained by a spherically-symmetric ejection from the Galactic Centre by the Hills mechanism. In Fig. 3 (a), we include a dashed line equivalent to $\delta=0^{\circ}$ which corresponds to the lower edge of the region of the sky which has been thoroughly searched for HVSs. The current searches for HVSs using SDSS are in the wrong part of the sky for the majority of an LMC escaping distribution. The other populations of OB stars shown in Fig. 3 are from comparatively shallow surveys down to magnitudes around $V=16 \mathrm{mag}$, while the known HVSs have SDSS magnitudes in the range $17.5<g_{0}<21.0$. If the observed HVS population does originate in the LMC, then the final Gaia catalogue, complete down to $G \approx 20.7 \mathrm{mag}$, could contain hundreds or even thousands of stars which have escaped the LMC, the majority of which would be HVSs.

\subsection{Observations of Outer LMC Populations}

Recently, Zhang et al. (2017) reported high-resolution spectra of eight previously claimed candidates (Casetti-Dinescu et al. 2012, 2014) for OB-type stars which have formed from the gas in the Leading Arm of the Magellanic System. They found that for five of these stars their chemistry was consistent with an LMC origin and that their kinematics appeared to rule out membership of the Milky Way disk. Zhang et al. (2017) concluded that these stars therefore must have formed from the gas in the leading arm. One property of these stars is however quite puzzling: none display a clear signal of radial velocity variation from a binary companion. Zhang et al. (2017) factor in the detection efficiency of their observations and calculate that the probability of their null detection is $14 \%(8.7 \%)$ if the underlying binary fraction is $50 \%(60 \%)$. While this is not statistically significant evidence for an unusually low binary fraction, the null detection of companions is entirely consistent with our prediction of B-type runaway stars from the LMC. Casetti-Dinescu et al. (2014) rejected a Galactic runaway origin for these five Btype stars arguing that their radial velocity dispersion of $33 \mathrm{~km} \mathrm{~s}^{-1}$ is too low compared to the $\sim 130 \mathrm{~km} \mathrm{~s}^{-1}$ (Bromley et al. 2009) expected for Milky Way runaways, and that an ejection mechanism would need to be "directionally coherent, which is highly unlikely". However, Casetti-Dinescu et al. (2014) do not consider a runaway origin from the LMC which naturally explains the low velocity dispersion. There is one O6V star, labelled by Zhang et al. (2017) as CD14-A08, which Casetti-Dinescu et al. (2014) do consider as originating in the LMC, but they argue it must have formed in-situ from the gas of the Leading Arm since its lifetime is too short (1-2 Myr) for it to have travelled from the LMC at any less than about $10^{4} \mathrm{~km} \mathrm{~s}^{-1}$. However, Zhang et al. (2017) argue that CD14-A08 is more likely to be a helium-deficient sdO star with $\log N_{\mathrm{He}} / N_{\mathrm{H}}=-1.69 \pm 0.24$. Martin et al. (2017) discuss the likely production mechanism of subdwarf stars as a function of their helium abundance. Helium-deficient subdwarfs are thought to be produced by close interactions in a binary and have ages between 0.2 and $10 \mathrm{Gyr}$, allowing CD14-A08 to have originated anywhere in the MW or the LMC. Martin et al. (2017) mention the possibility that intermediate-helium $\mathrm{sdO} / \mathrm{sdB}$ stars are the polluted, run- 
away companions of SN Ia progenitors, which has previously been used to explain the helium-rich HVS US708 (Justham et al. 2009; Geier et al. 2013). This suggests an intriguing alternative origin for CD14-A08 as a runaway from a Type Ia $\mathrm{SNe}$ in the LMC, which may be required if more precise data constrain the helium abundance to be in the range $5 \%<n_{\mathrm{He}}<80 \%$ considered by Martin et al. (2017) to be intermediate-helium. In Fig. 3, we show the kinematics of the Zhang et al. (2017) sample against the LMC runaway predictions. These stars are consistent with an LMC runaway origin. Their position near the edge of the LMC runaway distribution in radial velocity and distance is a natural consequence of the shallowness of the survey, which only probes the nearest edge of the distribution in regions where we would predict relatively low radial velocities.

Lennon et al. (2016) combined the precise proper motions of the Tycho Gaia Astrometric Solution (TGAS) with prior radial velocity surveys to search for runaway stars amongst the 31 brightest stars in the LMC. They found that only two of these 31 candidates are outliers in velocity, while the remaining stars are consistent with a rotating disk. In fact, the majority of our runaways would be classed as walkaways, with $65 \%$ of runaways having ejection velocities less than $10 \mathrm{~km} \mathrm{~s}^{-1}$, and hence indistinguishable from the disk population. There is also the statistical argument that most massive stars are in binaries, so most of these stars are either runaways or have a companion. Of the two outliers, Sk-67 2 is suggested as a candidate hypervelocity star based on a peculiar velocity of $359 \mathrm{~km} \mathrm{~s}^{-1}$ and $\mathrm{R} 71$ could be the evolved product of a slow runaway binary. Note that $R$ 71 is a Luminous Blue Variable (LBV). It was hypothesised by Smith \& Tombleson (2015) that the higher spatial dispersion of LBVs versus O-type and Wolf-Rayet stars in the LMC indicates either that LBVs are merged stars or they are runaway stars that were rejuvenated by mass transfer before being ejected. This contradicts the standard view of LBVs as a necessary transition state of massive stars between core hydrogen burning and the Wolf-Rayet phase. We seek analogues of the runaway candidates of Lennon et al. (2016) in our simulation, assuming they lie at a distance of $50.1 \pm 3.0 \mathrm{kpc}$, and find that most are consistent with a runaway origin (Fig. 3). We are hindered because we compare the brightest stars between observations and our model LMC runaway population. Small number statistics dominate and it is difficult to quantify whether any particular star is inconsistent with our model. The hypervelocity candidate Sk-67 2 is the clear outlier from the other candidates of Lennon et al. (2016) in Fig. 3 (d) where we plot $b-\mu_{\mathrm{b}}$. It is possible that the Hills mechanism or dynamical ejection is required to explain this star. The other outlier in Fig. 3 (c) is Sk-71 42 which Lennon et al. (2016) note as having a large astrometric_excess_noise parameter in TGAS and stated that further data would be necessary before they could speculate on the nature of the star.

It is interesting to note the similarities between Sk-67 2 and a previous discovery by Evans \& Massey (2015) of a 12-15 $\mathrm{M}_{\odot}$ runaway red supergiant J004330.06+405258.4 at a projected distance of $4.6 \mathrm{kpc}$ from the plane of M31's disk. Evans \& Massey (2015) mention that J004330.06+405258.4 may be a high-mass analogue of the MW HVSs since it is likely unbound from M31. Both stars are supergiants and both are discrepant with their host galaxies' kinematics by $\sim 300 \mathrm{~km} \mathrm{~s}^{-1}$. Evans \& Massey (2015) mention four previous discoveries of yellow and red supergiants in the LMC, SMC and M33 which have peculiar velocities around $150 \mathrm{~km} \mathrm{~s}^{-1}$. These massive runaways are difficult to reproduce in our current model, however a modification of the common-envelope prescription to produce more early-type stars would likely resolve this problem (Sec. 3.2). These stars are some of the brightest stars visible in the Local Group and so are obvious candidates for spectroscopic follow-up when they are found far from central star formation regions. It is possible that these stars are only the first tracers of a high-velocity runaway population which exists throughout the Local Group.

Bidin et al. (2017) searched for star formation on the periphery of the LMC disk between $6^{\circ}$ and $30^{\circ}$ from the centre. They found six recently formed stars well away from the central star formation in the LMC, with $V<16$, separation $7^{\circ}-13^{\circ}$ and ages between 10 and $50 \mathrm{Myr}$. They argued that if their tangential velocity is only as discrepant from the LMC disk tangential velocity as their radial velocity component, these stars cannot have travelled to their current location within their lifetimes. However, in our simulation, analogues of these stars do exist with similar ages because the assumption of equally discrepant velocity components does not hold. The existence of a ring-like structure is a natural consequence of sampling a small number of stars from a population which rapidly decreases in number with radius and is truncated at $6^{\circ}$ from the LMC.

\subsection{Prospects with Gaia}

The Gaia satellite is predicted to be complete down to $G \approx 20.7$, hence will be the first survey covering the Southern hemisphere which is sensitive to the population of runaway stars which may have escaped the LMC. We compare the predicted observable properties of the LMC runaways to the expected $\pm 1 \sigma$ end-of-mission radial velocity and proper motions errors for Gaia in Fig. 5. The proper motion errors are the predicted sky-average errors for an unreddened G2V star $^{1}$. The radial velocity errors are calculated for an unreddened G0V star using a standard performance model ${ }^{2}$ which is valid down to $G_{\mathrm{RVS}} \sim 16$, where we used the colour-colour relations calculated by Jordi et al. (2010) to convert $G$ to Johnson $V$ and $G_{\text {RVs }}$. The mean mass of the LMC escapers is $1.35 \mathrm{M}_{\odot}$ which justifies the choice of G0V/G2V to illustrate the errors, however there are a range of LMC escaper masses. More (less) massive stars will have larger (smaller) errors. The radial velocities measured by Gaia are unlikely to have the necessary precision to detect the population of escaping LMC runaways (Fig. 5 (a)), with the possible exception of the bright $G=15-16$ and fast $v_{r} \approx 500 \mathrm{~km} \mathrm{~s}^{-1}$ stars. Figures 5 (b) and (c) show that the $\mu$ as astrometric precision of Gaia should result in the detection of high velocity runaways purely by their proper motion. The uncertainties on the parallax measurements by Gaia rule out the possibility of a significant detection of parallax in LMC runaway stars. Distances would need to be obtained photometrically to validate any candidates. The LMC escapers will also be

\footnotetext{
1 https://www.cosmos.esa.int/web/gaia/sp-table1

2 https://www.cosmos.esa.int/web/gaia/

science-performance
} 
distinct from the LMC in their position on the sky and thus we conclude that Gaia will observe such a population if it exists. A change in the common envelope prescription to produce more early-type stars (Sec. 3.2) would not change this conclusion because the small increase in the astrometric uncertainties at fixed $G$ is more than cancelled by the shift of the distribution to brighter $G$ magnitudes.

\subsection{Exotica: Runaway Supernovae, Pulsars and Microlensing}

\subsubsection{Runaway Supernovae and Pulsars}

In our model, a substantial fraction of runaways (51.0\%) have experienced a core-collapse supernova before the present day, at a rate of $5.9 \times 10^{-4} \mathrm{yr}^{-1}$, leaving behind a compact neutron star or black hole remnant. The compact remnants experience a kick which we prescribe to be Maxwellian-distributed with a dispersion of $190 \mathrm{~km} \mathrm{~s}^{-1}$ (Hansen \& Phinney 1997). However, the fact that pulsars exist in globular clusters suggests that a fraction of neutron stars could receive almost no kick at birth (Podsiadlowski et al. 2005). Several authors have found that a bimodal Gaussian is required to describe the observed pulsar velocity distribution (Fryer et al. 1998; Cordes \& Chernoff 1998), but these studies differ on the required properties of such a distribution. Given that the runaway velocity distribution is itself uncertain, we feel justified in preferring the simplicity of a unimodal distribution in this study. The SN kick, in most cases, dominates the velocity of the remnant. The majority of these remnants subsequently escape the LMC and most of those are unbound from the Galaxy (Fig. 6). Despite the high kick dispersion, the distribution on the sky preserves the signal of their LMC origin and thus, if they are observable, their origin is unambiguous. There are few accessible observables associated with single, compact remnants at tens of kiloparsecs. However, for the first few tens of millions of years, neutron stars manifest themselves as pulsars.

The Australia Telescope National Facility Pulsar Catalogue (Manchester et al. 2005, available at http://www . atnf.csiro.au/research/pulsar/psrcat) reveals there are 29 pulsars currently associated with the LMC or SMC. We cannot accurately estimate the distance to these pulsars except through their plausible association with the Magellanic Clouds. For pulsars too far away for parallax measurements, the primary distance estimate is found by relating the dispersion measure to the integrated electron column density along the line of sight. This method is only reliable out to distances of $\sim 20 \mathrm{kpc}$. For example, the most recent electron density maps made by Yao et al. (2017) return a maximum distance of $25 \mathrm{kpc}$ to any pulsar with an anomalously high dispersion measure. There are 75 pulsars in our simulation closer than this upper limit. However, the completeness of the existing pulsar surveys is patchy at these distances, and all but one of the pulsars estimated to lie beyond $20 \mathrm{kpc}$ are in the direction of the well-studied Galactic bulge. The wide field of view and high sensitivity of the Square Kilometre Array will enable the discovery of 20,000 new pulsars (Smits et al. 2009). This is an order of magnitude increase in sample size and will provide a test of our model. The possibility that hundreds of thousands of neutron stars have

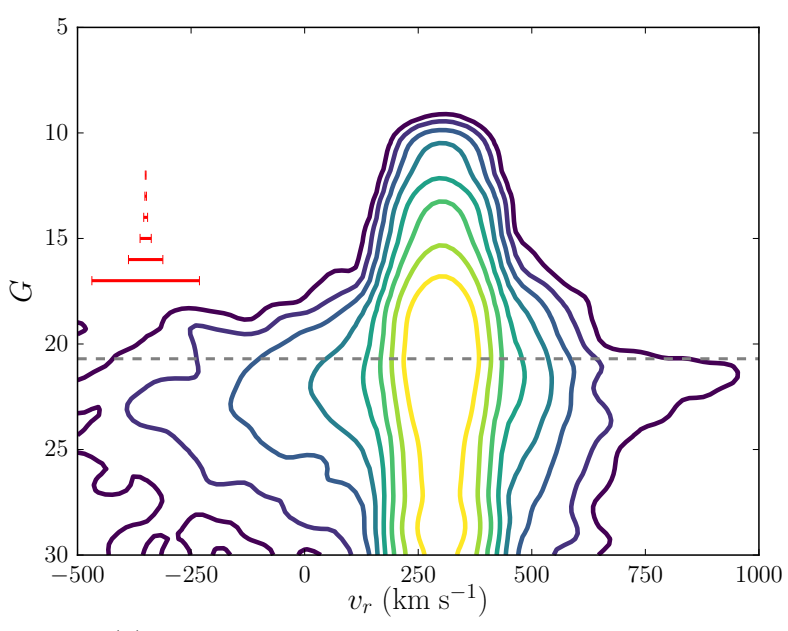

(a) Apparent Magnitude - Radial Velocity

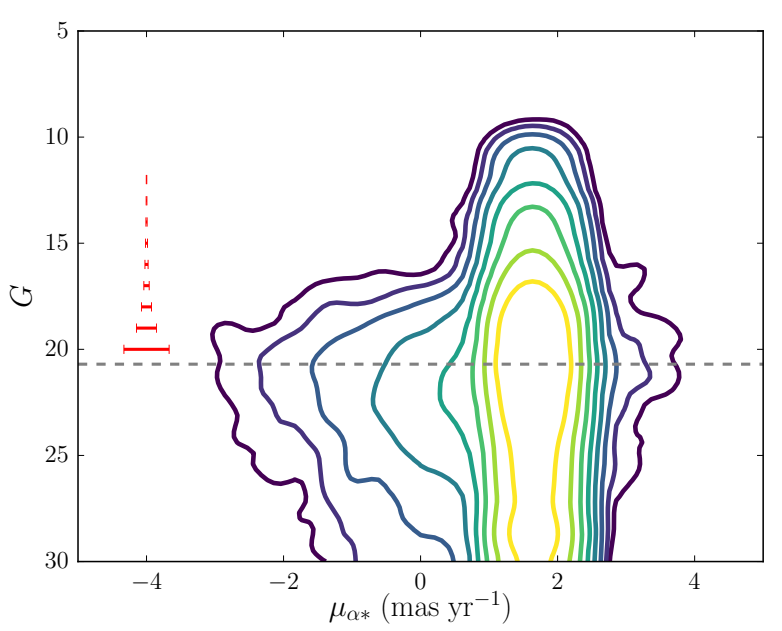

(b) Apparent Magnitude - Longitudinal Proper Motion

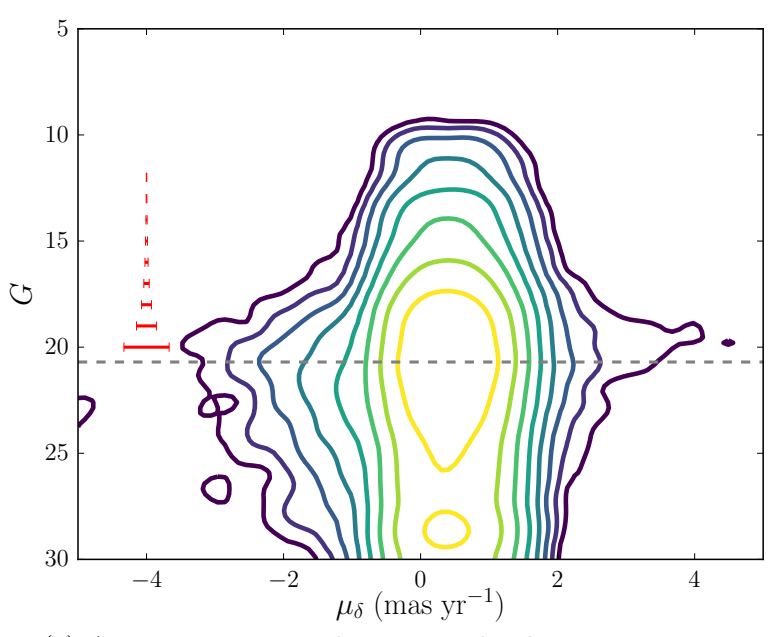

(c) Apparent Magnitude - Latitudinal Proper Motion

Figure 5. Predicted properties of LMC runaways which would be observed by Gaia plotted as logarithmically-spaced contours of the number of stars in each bin (see Fig. 3 for the colourbar). The kinematics are heliocentric and $G$ is the unreddened apparent magnitude. The grey dashed line indicates the $G \approx 20.7$ completeness limit for Gaia and the red error bars represent the $\pm 1 \sigma$ predicted end-of-mission radial velocity and proper motion errors as a function of $G$ (described in detail in Sec. 3.4). 


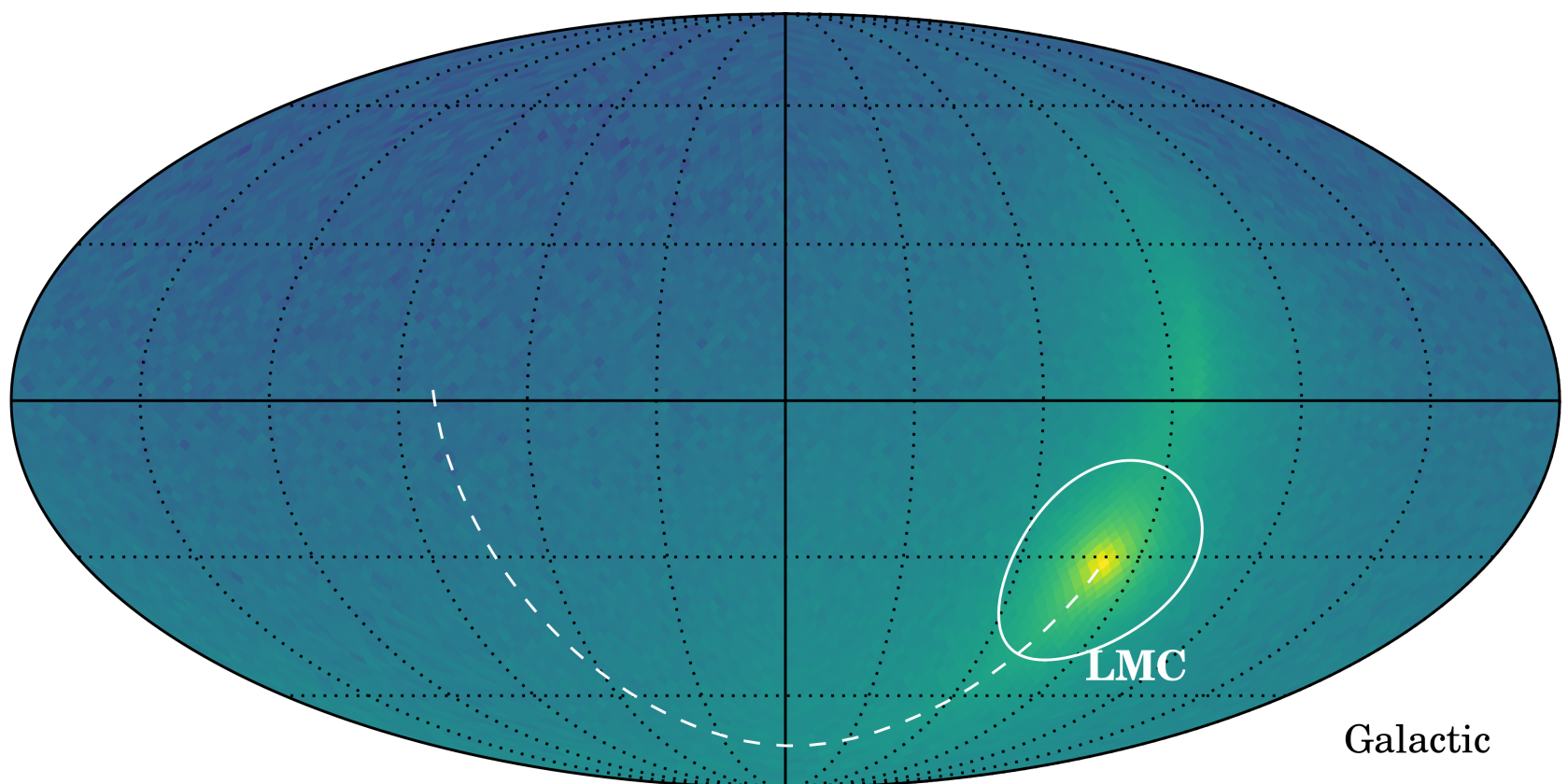

\section{Number of stars per HEALPix}

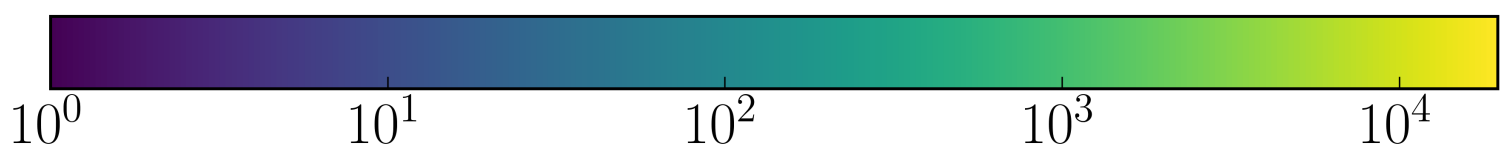

Figure 6. All sky distribution of remnants produced by runaway supernovae in our models (Sec. 3.5). The white solid line indicates the $20 \mathrm{kpc}$ tidal radius of the LMC and the white dashed line is the orbit of the LMC over the last 1.97 Gyr in the frame where the Sun is fixed at $(x, y, z)=\left(-R_{\odot}, 0,0\right)$.

been ejected from the LMC and are now populating the local IGM was mentioned by Ridley \& Lorimer (2010) in the context of single star evolution.

\subsubsection{Microlensing}

Photometric microlensing towards the LMC by an intervening population of dark objects was thought to be a straightforward test of the existence of massive compact halo objects (MACHOs), which may comprise some of the dark matter (Paczynski 1986). When the experiment was carried out, $40 \%$ of the microlensing optical depth was indeed unexplained by Galactic populations, such as the thick disk and halo. However, this signal is too small to be caused by MACHOs if they comprise the entirety of the Milky Way's dark matter halo. Several authors attempt to explain this excess with stellar populations at various points along the line of sight to the LMC (Zhao 1998; Evans \& Kerins 2000), though the viability of this explanation has also been disputed (Gould 1997, 1999). Besla et al. (2013) modelled the interaction of the LMC with the SMC and found that the microlensing might be explained by clumpy tidal debris from the SMC being microlensed by the LMC disk. Here, we consider whether our substantial population of neutron stars and black holes contributes to the microlensing optical depth to the LMC. We use the formula of Gould (1999) for the required surface mass density $\Sigma$ to contribute lensing optical depth $\tau_{\mathrm{p}}$,

$\Sigma=47\left(\frac{\tau_{\mathrm{p}}}{2.9 \times 10^{-7}}\right)\left(\frac{\hat{D}}{10 \mathrm{kpc}}\right)^{-1} \mathrm{M}_{\odot} \mathrm{pc}^{-2}, \quad \hat{D} \equiv \frac{d_{\mathrm{ol}} d_{\mathrm{ls}}}{d_{\mathrm{os}}}$,

where $d_{\mathrm{ol}}, d_{\mathrm{ls}}$ and $d_{\mathrm{os}}$ are the respective observer-lens, lenssource and observer-source distances. We find that our remnants contribute $0.0035 \%$ to the observed microlensing optical depth. In our calculations, we only include those remnants in front of the LMC and within three degrees of the sightline between the observer and the centre of the LMC.

Less familiar than photometric microlensing is the accompanying astrometric effect, in which the light centroid of the source is deflected by the presence of the foreground lens. Belokurov \& Evans (2002) calculated the all-sky photometric and astrometric microlensing optical depths detectable by Gaia and found that the astrometric optical depth was two orders of magnitude larger than the photometric optical depth. We calculate the astrometric optical depth $\tau_{\mathrm{a}}$ for our neutron star and black hole population using Equation 14 from Belokurov \& Evans (2002),

$\tau_{\mathrm{a}}=4 \sqrt{\frac{\mathrm{G}}{\mathrm{c}^{2}}} d_{\mathrm{os}}\left\langle M^{-1 / 2}\right\rangle \sqrt{\frac{T_{\text {life }}^{3} \nu^{3}}{5 \sqrt{2} \sigma_{\mathrm{a}}}} \int_{0}^{1} \rho(x) \sqrt{1-x} d x$,

where $\mathrm{G}$ is the gravitational constant, $\mathrm{c}$ is the speed of light, $\left\langle M^{-1 / 2}\right\rangle$ is the mean of the inverse square-root of the masses of the compact remnants, $T_{\text {life }}=5 \mathrm{yr}$ is the estimated lifetime of Gaia, $\sigma_{\mathrm{a}}=390 \mu$ as is the predicted mean position accuracy of Gaia for sources with $G=18 \mathrm{mag}, v \sim 140 \mathrm{~km} \mathrm{~s}^{-1}$ 
is a characteristic velocity of the lens relative to the LMC disk and $\rho(x)$ is the mass density at a fraction $x$ along the line-of-sight to the source. We find $\tau_{\mathrm{a}}=1.0 \times 10^{-10}$ which is 15 times greater than the corresponding photometric microlensing optical depth. However, this optical depth is likely still too small to give observable consequences.

\section{CONCLUSIONS}

We have presented a novel source of hypervelocity stars (HVSs) in the Milky Way (MW) halo. In our model, HVSs originate as runaway stars from the Large Magellanic Cloud (LMC). The known HVSs possess the kinematics expected of stars which have been ejected from the LMC, and thus an LMC origin for some of these stars must be considered a realistic possibility.

There are a number of current observations that support our scenario, albeit indirectly. This includes: (i) a sample of the 31 brightest stars in the LMC which are consistent with runaway expectations except perhaps from one anomalously fast supergiant (Lennon et al. 2016), (ii) young stars in the periphery of the LMC far from star formation regions (Bidin et al. 2017) and (iii) B-type stars in the gaseous leading arm of the LMC with LMC kinematics and chemistry whose anomalous single nature is in line with a runaway origin (Zhang et al. 2017).

The HVSs found in the Sloan Digital Sky Survey footprint are B-type stars with masses exceeding $3 \mathrm{M}_{\odot}$. In our model, the LMC runaways that end up as hypervelocity in the Sloan footprint have somewhat smaller masses, typically between $1.6 \mathrm{M}_{\odot}<M<3.0 \mathrm{M}_{\odot}$. However, there is a strong dependency of the mass and colour of the produced HVSs on the common-envelope prescription, with lower common-envelope ejection efficiencies broadly associated with higher mass hypervelocity stars. So, this discrepancy could be resolved by modest changes to the uncertain prescription of common envelope evolution. Alternatively, the observed HVS population may have contributions from multiple processes only one of which is the fast moving LMC runaway stars.

Our model leads to predictions of the spatial and kinematic signatures of HVSs seen by Gaia and the hypervelocity pulsars observed by the Square Kilometre Array. We predict that both will be preferentially found along the past and future orbit of the LMC. The final Gaia catalogue aims to be complete down to $G \approx 20.7$ subject to crowding in dense fields. This should detect a large number of hypervelocity runaways from the LMC. We would expect about 200 of these stars at distances $30<d<120 \mathrm{kpc}$ and with proper motions around 1 mas. This corresponds to a (heliocentric) tangential velocity of around $500 \mathrm{~km} \mathrm{~s}^{-1}$ at the location of the LMC. However, we do not expect either parallax or radial velocities for these stars from Gaia, so identification of their nature will rely on photometric distances and spectroscopy.

In investigating the runaway processes in the LMC, we have linked a binary stellar evolution code with an N-body model of the interaction between the Galaxy and the LMC, which enabled us to make powerful predictions. A problem which required bringing together stellar evolution and stellar dynamics has implications for both. LMC runaway stars can provide important constraints on both commonenvelope dispersal and the escape velocity of the Milky Way.

Elsewhere, we have argued that a super-massive black hole (SMBH) in the LMC may generate HVSs by the Hills mechanism (Boubert \& Evans 2016). This remains plausible, though evidence for an SMBH in the LMC is elusive at present. However, runaway stars are a natural consequence of binary evolution in a star-forming galaxy, and hence they will certainly exist in the LMC. The exceptionally fast runaways, which become HVSs with respect to the Milky Way, are sensitive to the prescription of binary evolution. Changing the binary evolution only seems to modify the properties of those HVSs and not their number or distribution on the sky. Our argument therefore does not rely on the precise details of binary evolution. Furthermore, there are observed counterparts to our evolutionary channel. A pulsar - helium white dwarf binary is left behind if the system is not unbound during the supernova, but is close enough after the end of common-envelope evolution that the companion is stripped before igniting helium. The extreme velocity of the runaways originates in the orbital velocity of such close binaries. We conclude that hypervelocity runaway stars from the LMC, as a consequence of star-formation, are unavoidable. They must contribute to the Galactic HVS population. The only argument is whether this process is dominant or subordinate.

\section{ACKNOWLEDGEMENTS}

The authors would like to thank the anonymous reviewer whose suggestion of a plot of apparent magnitude and radial velocity improved the usefulness of our prediction for observers. The authors thank Mathieu Renzo, Simon Stevenson, Manos Zapartas and the many other authors cited above who have contributed to the development of BINARY_C. We also thank Vasily Belokurov and other members of the Streams discussion group for comments on this work as it was under development. DB thanks Isaac Shivers for a thought-provoking visualisation of the Galactic pulsar distribution (http://w.astro.berkeley.edu/ ishivvers/ pulsars.html). DB is grateful to the Science and Technology Facilities Council (STFC) for providing PhD funding. DE acknowledges that research leading to these results has received funding from the European Research Council under the European Union's Seventh Framework Programme (FP/2007-2013)/ERC Grant Agreement no. 308024. RGI thanks the STFC for funding his Rutherford fellowship under grant ST/L003910/1 and Churchill College, Cambridge for his fellowship.

\section{REFERENCES}

Abadi M. G., Navarro J. F., Steinmetz M., 2009, ApJL, 691, L63 Abate C., Pols O. R., Izzard R. G., Mohamed S. S., de Mink S. E., 2013, A\&A, 552, A26

Abate C., Pols O. R., Stancliffe R. J., Izzard R. G., Karakas A. I., Beers T. C., Lee Y. S., 2015, A\&A, 581, A62

Althaus L. G., Benvenuto O. G., 1997, ApJ, 477, 313

Arenou F., 2010, The simulated multiple stars, www.rssd.esa. int/doc_fetch.php?id=2969346

Backer D. C., 1998, ApJ, 493, 873 
Belokurov V. A., Evans N. W., 2002, MNRAS, 331, 649

Besla G., Hernquist L., Loeb A., 2013, MNRAS, 428, 2342

Bidin C. M., Casetti-Dinescu D. I., Girard T. M., Zhang L., Mendez R. A., Vieira K., Korchagin V. I., van Altena W. F., 2017, MNRAS, 466, 3077

Blaauw A., 1961, Bulletin of the Astronomical Institutes of the Netherlands, 15, 265

Boubert D., Evans N. W., 2016, ApJL, 825, L6

Bromley B. C., Kenyon S. J., Brown W. R., Geller M. J., 2009, ApJ, 706, 925

Brown W. R., 2015, ARA\&A, 53, 15

Brown W. R., Geller M. J., Kenyon S. J., Kurtz M. J., 2005, ApJL, 622, L33

Brown W. R., Geller M. J., Kenyon S. J., Kurtz M. J., 2006a, ApJL, 640, L35

Brown W. R., Geller M. J., Kenyon S. J., Kurtz M. J., 2006b, ApJ, 647, 303

Brown W. R., Geller M. J., Kenyon S. J., Kurtz M. J., Bromley B. C., 2007a, ApJ, 660, 311

Brown W. R., Geller M. J., Kenyon S. J., Kurtz M. J., Bromley B. C., 2007b, ApJ, 671, 1708

Brown W. R., Geller M. J., Kenyon S. J., Bromley B. C., 2009a, ApJL, 690, L69

Brown W. R., Geller M. J., Kenyon S. J., 2009b, ApJ, 690, 1639

Brown W. R., Geller M. J., Kenyon S. J., 2012, ApJ, 751, 55

Brown W. R., Geller M. J., Kenyon S. J., 2014, ApJ, 787, 89

Brown W. R., Anderson J., Gnedin O. Y., Bond H. E., Geller M. J., Kenyon S. J., 2015, ApJ, 804, 49

Casetti-Dinescu D. I., Vieira K., Girard T. M., van Altena W. F., 2012, ApJ, 753, 123

Casetti-Dinescu D. I., Bidin C. M., Girard T. M., Mendez R. A., Vieira K., Korchagin V. I., van Altena W. F., 2014, ApJ, 784, L37

Cignoni M., et al., 2015, ApJ, 811, 76

Claeys J. S. W., Pols O. R., Izzard R. G., Vink J., Verbunt F. W. M., 2014, A\&A, 563, A83

Cordes J. M., Chernoff D. F., 1998, ApJ, 505, 315

De Marchi G., et al., 2011, ApJ, 739, 27

De Marco O., Izzard R. G., 2017, PASA, 34, e001

de Mink S. E., Brott I., Cantiello M., Izzard R. G., Langer N., Sana H., 2012. Challenges for Understanding the Evolution of Massive Stars: Rotation, Binarity, and Mergers, Proceedings of a Scientific Meeting in Honor of Anthony F. J. Moffat, p. 65

de Mink S. E., Langer N., Izzard R. G., Sana H., de Koter A., 2013, ApJ, 764, 166

de Mink S. E., Sana H., Langer N., Izzard R. G., Schneider F. R. N., 2014, ApJ, 782, 7

Dewi J. D. M., Tauris T. M., 2000, A\&A, 360, 1043

Duquennoy A., Mayor M., 1991, A\&A, 248, 485

Edelmann H., Napiwotzki R., Heber U., Christlieb N., Reimers D., 2005, ApJL, 634, L181

Evans N. W., Kerins E., 2000, ApJ, 529, 917

Evans K. A., Massey P., 2015, AJ, 150, 149

Fryer C., Burrows A., Benz W., 1998, ApJ, 496, 333

Geier S., et al., 2013, A\&A, 554, A54

Gould A., 1997, preprint (arXiv:astro-ph/9709263)

Gould A., 1999, ApJ, 525, 734

Gualandris A., Portegies Zwart S., 2007, MNRAS, 376, L29

Hansen B. M. S., Phinney E. S., 1997, MNRAS, 291, 569

Harris J., Zaritsky D., 2009, AJ, 138, 1243

Hills J. G., 1988, Nature, 331, 687

Hirsch H. A., Heber U., O'Toole S. J., Bresolin F., 2005, A\&A, 444, L61

Hurley J. R., Tout C. A., Pols O. R., 2002, MNRAS, 329, 897

Kallivayalil N., van der Marel R. P., Besla G., Anderson J., Alcock C., 2013, ApJ, 764, 161

Izzard R. G., Tout C. A., Karakas A. I., Pols O. R., 2004, MNRAS, 350,407

MNRAS 000, 1-13 (2017)
Izzard R. G., Dray L. M., Karakas A. I., Lugaro M., Tout C. A., 2006, A\&A, 460, 565

Izzard R. G., Glebbeek E., Stancliffe R. J., Pols O. R., 2009, A\&A, 508, 1359

Jethwa P., Erkal D., Belokurov V., 2016, MNRAS, 461, 2212

Jordi C., et al., 2010, A\&A, 523, A48

Justham S., Wolf C., Podsiadlowski P., Han Z., 2009, A\&A, 493, 1081

Kenyon S. J., Bromley B. C., Brown W. R., Geller M. J., 2014, ApJ, 793, 122

Kroupa P., 2001, MNRAS, 322, 231

Lada C. J., Lada E. A., 2003, ARA\&A, 41, 57

Lennon D. J., van der Marel R. P., Lerate M. R., O'Mullane W., Sahlmann J., 2016, preprint (arXiv:1611.05504)

Liu Z.-W., Tauris T. M., Röpke F. K., Moriya T. J., Kruckow M., Stancliffe R. J., Izzard R. G., 2015, A\&A, 584, A11

Mackey A. D., Koposov S. E., Erkal D., Belokurov V., Da Costa G. S., Gomez F. A., 2016, MNRAS, 459, 239

Manchester R. N., Hobbs G. B., Teoh A., Hobbs M., 2005, AJ, 129, 1993

Martin P., Jeffery C. S., Naslim N., Woolf V. M., 2017, preprint (arXiv:1701.03026)

Opik E., 1924, Publications of the Tartu Astrofizica Observatory, 25

Paczynski B., 1986, ApJ, 304, 1

Peñarrubia J., Gómez F. A., Besla G., Erkal D., Ma Y.-Z., 2016, MNRAS, 456, L54

Perets H. B., 2009, ApJ, 690, 795

Perets H. B., Šbr L., 2012, ApJ, 751, 133

Piatti A. E., Geisler D., 2013, AJ, 145, 17

Podsiadlowski P., Pfahl E., Rappaport S., 2005. Binary Radio Pulsars, Astronomical Society of the Pacific Conference Series, p. 327

Portegies Zwart S. F., 2000, ApJ, 544, 437

Przybilla N., Nieva M. F., Heber U., Firnstein M., Butler K., Napiwotzki R., Edelmann H., 2008, A\&A, 480, L37

Raghavan D., et al., 2010, ApJS, 190, 1

Ridley J. P., Lorimer D. R., 2010, MNRAS, 406, L80

Sana H., et al., 2012, Science, 337, 444

Schlegel D. J., Finkbeiner D. P., Davis M., 1998, ApJ, 500, 525

Schneider F. R. N., et al., 2014, ApJ, 780, 117

Schönrich R., Binney J., Dehnen W., 2010, MNRAS, 403, 1829

Smith N., Tombleson R., 2015, MNRAS, 447, 598

Smits R., Kramer M., Stappers B., Lorimer D. R., Cordes J., Faulkner A., 2009, A\&A, 493, 1161

Spera M., Mapelli M., Bressan A., 2015, MNRAS, 451, 4086

Springel V., 2005, MNRAS, 364, 1105

Tauris T. M., Dewi J. D. M., 2001, A\&A, 369, 170

Tauris T. M., Takens R. J., 1998, A\&A, 330, 1047

van der Marel R. P., Kallivayalil N., 2014, ApJ, 781, 121

Yao J. M., Manchester R. N., Wang N., 2017, ApJ, 835, 29

Zapartas E., et al., 2017, preprint (arXiv:1701.07032)

Zhang L., et al., 2017, preprint (arXiv:1701.00590)

Zhao H., 1998, MNRAS, 294, 139

This paper has been typeset from a $\mathrm{T}_{\mathrm{E}} \mathrm{X} / \mathrm{LAT} \mathrm{TX}$ file prepared by the author. 